\title{
Mucinous cystadenoma of appendix
}

\author{
Sanjay Gupta $\cdot$ Rajeev Sharma $\cdot$ Ashok K. Attri $\cdot$ Ravinder Kaur
}

Received: 9 July 2008 / Accepted: 14 September 2008

The mucocele of the appendix is rare occurrence, with reported incidence of $0.2-0.3 \%$ among all the appendecectomy specimens [1]. A variety of underlying neoplastic and non neoplastic pathological changes can lead to appendicular mucocele. The size of the mucocele helps in predicting the nature of the cyst. Cysts less than $2 \mathrm{~cm}$ in diameter are usually simple retention cysts with normal epithelium, whereas those more than $2 \mathrm{~cm}$ may occur because of hyperplastic epithelium, cystadenoma or cystadenocarcinoma [2]. One such type of mucocele is reported and the condition is briefly reviewed.

A 46-yrs-old male presented with firm, mobile mass in the right iliac fossa for 2 months, associated with mild pain. There was no history suggestive of bowel pathology or past attack of appendicitis. His routine investigations were within normal limits. CECT of the abdomen revealed well encapsulated, hypo attenuated mass in the region of the appendix (Fig. 1). His colonoscopic examination was normal. On exploration, appendix was found to be grossly enlarged $(10 \times 5 \mathrm{~cm})$, having cystic consistency (Fig. 2). A limited right hemicolectomy with end to end anastomosis was performed. Post operative period was uneventful. Gross pathological examination revealed distended appendix filled with mucin. On microscopic examination, appendiceal cyst was lined by single layer of columnar cells having apical mucosal vacuoles. There was no evidence of epithelial atypia or

S. Gupta $\cdot$ R. Sharma $\cdot$ A. K. Attri $\cdot$ R. Kaur

${ }^{1}$ Department of Surgery,

${ }^{2}$ Department of Radiodiagnosis,

Government Medical College \& Hospital,

Chandigarh, India

S. Gupta $(\square)$

e-mail: sandiv99@yahoo.co.uk invasion. It was labelled as mucinous cystadenoma of the appendix.

Mucinous Cystadenomas (63-84\%), are most common cause of mucoceles of the appendix [2]. Histopathologically, these have epithelium similar to that of villous adenoma or adenomatous polyp of the colon. Also the chances of progression to cystadenocarcinoma are similar to that seen with colonic adenomas [3]. Chronic right lower abdominal pain secondary to the cystic dilatation of the appendix is the most common presentation, followed by palpable mass. Among the various available diagnostic modalities, CECT of the abdomen is most useful in diagnosing the cystic neoplasms of the appendix. A hypoattenuated cystic mass in the appendiceal region with curvilinear mural calcifications, irregular wall thickening, or both, is considered the most specific CT feature of mucocele. Sometimes sonogra-

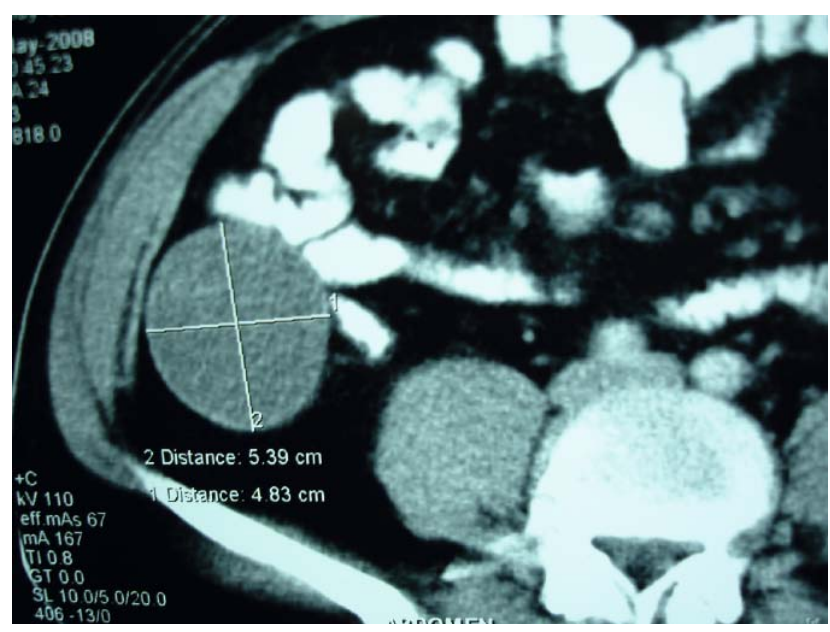

Fig. 1 CECT: Hypoattenuated, encapsulated mass in the region of the appendix. 


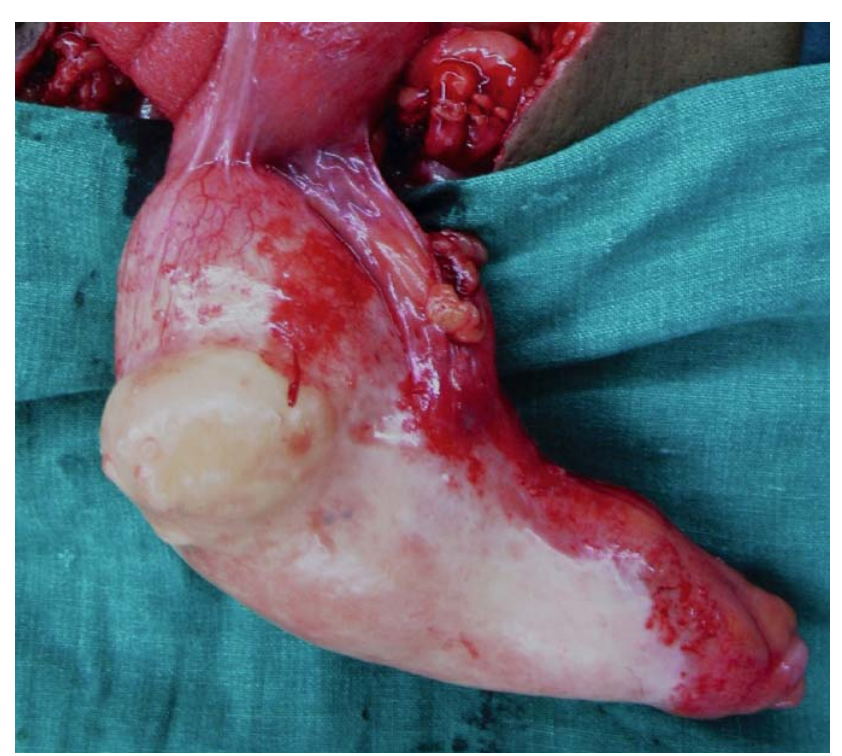

Fig. 2 Per operative picture showing distended appendix.

phy is more informative than CT scan if typical 'onion skin' sign due to wavy pattern of dense mucoid material inside the lesion, is seen [4]. Differential diagnosis of mucocele is from mesenteric cyst, omental cyst, hematoma, abscess and ovarian cyst in the females. Combined findings of low density mass at $\mathrm{CT}$ and cystic mass with echogenic foci at sonography in the region of appendix, are helpful in differentiating from other lesions [5].

Pre operative histopathological confirmation is unusual as FNAC of the cystic lesions of the appendix is avoided because of fear of implantation of neoplastic cells on the intraperitoneal surface leading to localised or diffuse pseudomyxoma peritonei. Simple appendecectomy is sufficient for the mucoceles less than $2 \mathrm{~cm}$ in diameter provided the base is free of disease whereas limited or partial right hemicolectomy is advocated for the lesions greater than $2 \mathrm{~cm}$ in diameter or when the base with the caecum is involved [3]. The entire mesoappendix should be excised as part of the specimen if malignancy is suspected. A formal right hemicolectomy should be avoided as this does not offer any survival advantage. Moreover, implantation of the cells in the exposed reteroperitoneum has been found to decrease survival and is difficult to treat. It should be reserved for the patients with positive appendiceal and distal ileo-colic lymph nodes, or an inadequate margin of resection [6]. Conventional surgery is preferred rather than laparoscopic approach because of associated risk of rupture and subsequent pseudomyxoma peritonei [1]. As associated colonic neoplasm may be present surveillance colonscopy is also recommended in the follow-up [3].

\section{Refrences}

1. Rampone B, Roviello F, Marrelli D, Pinto E (2005) Giant appendiceal mucocele: report of a case and brief review. World J Gastroenterol 11:4761-4763

2. Pickhardt PJ, Levy AD, Rohrmann CA, Kende AI (2003) Primary neoplasms of the appendix: radiologic spectrum of disease with pathologic correlation. RadioGraphics 23:645-662

3. Stocchi L, Wolff B, Larson D, Harrington J (2003) Surgical treatment of appendiceal mucocele. Arch Surg 138:585-589

4. Horgan JG, Chow PP, Richter JO, Rosenfield AT, Taylor KJ (1984) CT and sonography in the recognition of mucocele of the appendix. Am J Roentgenol 143(5):959-962

5. Francica G, Lapiccirella G, GiardielloC, Scarno F, Angelone G, De Marino F, Molese V (2006) Giant mucocele of the appendix: clinical and imaging findings in 3 cases. J Ultrasound Med 25(5):643-648

6.. Gonzalez-Moreno S, Sugarbaker PH (2004) Right hemicolectomy does not confer a survival advantage in patients with mucinous carcinoma of the appendix and peritoneal seeding. Br J Surg 91:304-311 